\title{
Impaired PLP-dependent metabolism in brain samples from Huntington disease patients and transgenic R6/1 mice
}

\author{
M. Alba Sorolla ${ }^{1}$ - María José Rodríguez-Colman ${ }^{1} \cdot$ Núria Vall-llaura $^{1} \cdot$ Celia Vived $^{1}$ \\ Marta Fernández-Nogales ${ }^{2}$ • José J. Lucas ${ }^{2} \cdot$ Isidre Ferrer $^{3} \cdot$ Elisa Cabiscol $^{1}$
}

Received: 1 September 2015 / Accepted: 9 December 2015

(C) Springer Science+Business Media New York 2015

\begin{abstract}
Oxidative stress has been described as important to Huntington disease (HD) progression. In a previous HD study, we identified several carbonylated proteins, including pyridoxal kinase and antiquitin, both of which are involved in the metabolism of pyridoxal 5'-phosphate (PLP), the active form of vitamin B6. In the present study, pyridoxal kinase levels were quantified and showed to be decreased both in HD patients and a R6/1 mouse model, compared to control samples. A metabolomic analysis was used to analyze metabolites in brain samples of HD patients and R6/1 mice, compared to control samples using mass spectrometry. This technique allowed detection of increased concentrations of pyridoxal, the substrate of pyridoxal kinase. In addition, PLP, the product of the reaction, was decreased in striatum from R6/1 mice. Furthermore, glutamate and cystathionine, both substrates of PLP-dependent enzymes were increased in HD. This reinforces the hypothesis that PLP synthesis is impaired, and could explain some alterations observed in the disease. Together, these results identify PLP as a potential therapeutic agent.
\end{abstract}

M. Alba Sorolla

alba.sorolla@cmb.udl.cat

1 Departament de Ciències Mèdiques Bàsiques, Universitat de Lleida, IRBLleida, Av. Rovira Roure 80, 25198 Lleida, Catalonia, Spain

2 Centro de Biología Molecular "Severo Ochoa", Consejo Superior de Investigaciones Científicas, Universidad Autónoma de Madrid, Madrid, Spain

3 Institut de Neuropatologia, Servei Anatomia Patològica, IDIBELL-Hospital Universitari de Bellvitge, Universitat de Barcelona, Barcelona, Spain
Keywords Huntington disease $\cdot$ Oxidative stress $\cdot$ Pyridoxal kinase $\cdot$ Pyridoxal 5-phosphate $\cdot$ Glutamate $\cdot$ Cystathionine

\begin{tabular}{|c|c|}
\hline \multicolumn{2}{|c|}{ Abbreviations } \\
\hline ALDH7A1 & $\begin{array}{l}\alpha \text {-aminoadipic semialdehyde dehydrogenase or } \\
\text { antiquitin }\end{array}$ \\
\hline GSH & Reduced glutathione \\
\hline HD & Huntington disease \\
\hline $\mathrm{Htt}$ & Huntingtin protein \\
\hline $\mathrm{mHtt}$ & Mutant huntingtin \\
\hline $\mathrm{LC}$ & Liquid chromatography \\
\hline MOPS & 3-(N-Morpholino)propanesulfonic acid \\
\hline MS & Mass spectrometry \\
\hline PDXK & Pyridoxal kinase \\
\hline PLP & Pyridoxal 5'-phosphate \\
\hline TOF & Time of flight \\
\hline
\end{tabular}

\section{Introduction}

Huntington disease (HD) is a dominantly inherited neurodegenerative disease caused by the expansion of CAG trinucleotide repeats in exon 1 of the huntingtin gene that encodes a stretch of polyglutamines (polyQ) in the huntingtin (Htt) protein (Group 1993). The disease appears when the number of glutamines exceeds 40. Age of onset and disease severity are strongly correlated with the length of the CAG expansion (Benitez et al. 1994). Clinical symptoms include psychiatric and cognitive abnormalities, as well as involuntary movements (chorea). With HD progression, motor rigidity, depression, and dementia predominate, and inevitably lead to death. Anatomically, HD is characterized by progressive brain atrophy, affecting predominantly the striatum, and the cortex to a lesser extent (Reiner et al. 1988). Htt is a widely expressed 
350-kDa protein that has been described to be related to several roles, however, its function is still unknown. One of the most significant findings in HD neuropathology was the presence of mutant $\mathrm{Htt}(\mathrm{mHtt})$ deposits, mostly in striatum and cortex (DiFiglia et al. 1997). Although the presence of aggregates often correlates with toxicity (Perutz 1999), it has been described that the oligomeric species, formed before the constitution of the mature $\mathrm{mHtt}$ deposits, are highly toxic and responsible for the cellular alterations observed in HD (Arrasate et al. 2004). Increasing evidence supports the involvement of mitochondria in HD pathogenesis. Impaired energy metabolism leading to ATP depletion, due to functional defects in mitochondrial complexes (Jenkins et al. 1993; Shirendeb et al. 2011) suggest that defective mitochondria may be responsible for neuronal damage in HD. Such mitochondrial damage might generate an oxidative stress situation well described in HD (Dugan et al. 1995; Browne 2008; Sorolla et al. 2008). Thus, cellular dysfunction in HD has been related to loss of normal physiological function and especially to gain of toxic function of $\mathrm{mHtt}$.

In previous publications using proteomic techniques (Sorolla et al. 2008; Sorolla et al. 2010), we demonstrated in human HD samples and also in a mouse model that several enzymes were oxidized (carbonylated) in striatum. These results pointed to a possible disturbance in the metabolism of pyridoxal 5'-phosphate (PLP), the active form of vitamin B6. Two enzymes, pyridoxal kinase (PDXK) and antiquitin 1, were identified as carbonylated proteins in human HD and the conditional HD mouse model Tet/HD94 (Sorolla et al. 2010). PDXK phosphorylates pyridoxal generating PLP. Antiquitin 1, encoded by the $A L D H 7 A 1$ gene, acts as a $\delta-1-$ piperideine-6-carboxylate dehydrogenase or $\alpha$-aminoadipic semialdehyde dehydrogenase. Mutations in the ALDH7A1 gene cause pyridoxine-dependent seizures, a disease affecting newborns and children. In these cases, mutations abolish antiquitin 1 activity and the accumulating substrate, piperideine-6-carboxylate, inactivates PLP by forming a Knoevenagel condensation product (Mills et al. 2006). The lack of PLP causes seizures and is associated with learning difficulties. These seizures are fully controlled by pyridoxine (Rankin et al. 2007). Thus, antiquitin 1 oxidation observed in HD could result in its inactivation, exacerbating the lack of PLP available as a cofactor. The active form of vitamin B6 is a necessary cofactor in more than 140 distinct enzymatic activities catalogued by the Enzyme Commission (http://www. chem.qmul.ac.uk/iubmb/enzyme/). To study whether such enzymes might be affected in HD, the substrate of two key PLP-dependent enzymes, glutamate and cystathionine, were analyzed. They are involved in the synthesis of GABA, a neurotransmitter described to be decreased in striatal neurons of HD patients and, ii) synthesis of glutathione (GSH), a key molecule maintaining the cellular redox status and the GSHdependent antioxidant defense system.
In the present study, we investigated the hypothesis that metabolic changes resulting from oxidation of PDXK and antiquitin are involved in HD pathogenesis due to decreased PLP levels. We report decreased levels of PDXK, both in HD patients and R6/1 transgenic mice. Using LC-MS metabolomics several metabolites were quantified giving support to an impaired PLP synthesis and, as a consequence, an altered PLP-dependent metabolism in HD. The biological significance in HD pathology is discussed.

\section{Material and methods}

\section{Human samples}

Human brain tissues (striatum and cortex) were obtained from the Institute of Neuropathology and University of Barcelona Brain Banks (Barcelona, Spain), following the guidelines of the local ethics committees. Control cases had not suffered neurologic or psychiatric disease and the neuropathology examination was strictly normal, including lack of Alzheimer disease-associated changes. Eight HD cases (range 41-62 CAG repeats) and eight controls were analyzed (Table 1). Control and HD groups were balanced for age, sex and postmortem time, excluding these parameters as potential differences between these groups. After neurological examination, all HD cases were classified as stage 4 according to Vonsattel et al. (1985). All procedures were carried out according to the Helsinki declaration and conducted according to the guidelines of the European Community Council.

Table 1 Summary of controls (C) and HD (H) cases

\begin{tabular}{llll}
\hline Patient & Sex & Age & Postmortem delay (h) \\
\hline C1 & Female & 73 & 7 \\
C2 & Female & 73 & 5.5 \\
C3 & Male & 51 & 4 \\
C4 & Female & 24 & 6 \\
C5 & Male & 70 & 13 \\
C6 & Female & 47 & 9.6 \\
C7 & Male & 53 & 3 \\
C8 & Male & 46 & 15 \\
H1 & Female & 72 & 7 \\
H2 & Female & 72 & 5 \\
H3 & Male & 68 & 4 \\
H4 & Female & 28 & 4.2 \\
H5 & Male & 71 & 10.2 \\
H6 & Female & 65 & 15.2 \\
H7 & Male & 59 & 5.5 \\
H8 & Male & 60 & 13.1 \\
\hline
\end{tabular}




\section{Murine samples}

Murine brain tissues (striatum, cortex and cerebellum) were obtained from wild-type (WT) and R6/1 transgenic mice for the human exon-1-Htt gene (CAG repeats: 168-189), all of them in B6CBAF1/J background (Mangiarini et al. 1996). Either 3-month-old ( $n=7$, each group) and 7-month-old ( $n=4$, each group) mice were bred at Severo Ochoa Center (Madrid). Procedures using laboratory animals were in accordance with the European Union Directives and institutional guidelines approved by the research ethics committee of Consejo Superior de Investigaciones Científicas (CSIC). All efforts were made to minimize the number of animals used and the suffering involved. Mice were maintained in a temperature-controlled environment on a 12-h light/dark cycle with food and water ad libitum.

\section{Sample preparation}

To perform Western blot analysis, brain tissue was homogenized as described (Sorolla et al. 2010). Tissue extracts were separated by SDS-PAGE and transferred to polyvinylidene difluoride membranes. The following antibodies were used: anti-PDXK (Santa Cruz Biotechnology; Ref. sc-67,361), antiantiquitin (Santa Cruz Biotechnology; Ref. SC79399), and anti-actin (Sigma; Ref. A1978). Images from Western blots were captured using a Chemidoc XRS system (BioRad) and band intensities were quantified by Quantity One software (BioRad). In metabolomic studies, brain tissue $(10 \mathrm{mg})$ was homogenized in a $200 \mu \mathrm{l}$ buffer containing $1 \mathrm{mM}$ MOPS, $180 \mathrm{mM} \mathrm{KCl}$, and $2 \mathrm{mM}$ EDTA, plus $1 \mathrm{mM}$ diethylenetriaminepentaacetic acid (DTPAC) as reductant and $1 \mu \mathrm{M}$ butylated hydroxytoluene (BHT) as antioxidant. The protein was precipitated with cold methanol and incubation for $60 \mathrm{~min}$ at $-20^{\circ} \mathrm{C}$. The sample was centrifuged at 12 , $000 \times g$ for $5 \mathrm{~min}$ to remove protein. The supernatant was dried in a vacuum centrifuge (Speed-Vac), resuspended in $100 \mu \mathrm{l}$ water and filtered using microcentrifuge filters.

\section{Data acquisition and analysis}

The system used was an HPLC 1200 series coupled to an ESIQ-TOF - MS/MS 6520 (Agilent Technologies). The deproteinized extract $(4 \mu \mathrm{l})$ was applied to a reverse phase column (C18 Luna $3 \mathrm{n} \mathrm{pfp(2)} 100 \AA 150 \times 2 \mathrm{~mm}$, Phenomenex). The flow rate was $200 \mu \mathrm{L} / \mathrm{min}$. Solvent A was composed of water containing $0.1 \%$ formic acid. Solvent B was composed of $95 \%$ acetonitrile and $5 \%$ water containing $0.1 \%$ formic acid. A 20 -min linear gradient ranging from 5 to $100 \%$ solvent B was performed. Data were collected in positive electrospray mode TOF (time of flight), operated in full-scan mode at 100 to $3000 \mathrm{~m} / \mathrm{z}$ in an extended dynamic range (2 GHz), using $\mathrm{N} 2$ as the nebulizer gas $(5 \mathrm{~L} /$ $\left.\min , 350{ }^{\circ} \mathrm{C}\right)$. The capillary voltage was $3500 \mathrm{~V}$ with a scan rate of $1 \mathrm{scan} / \mathrm{s}$.

Data Analysis and Quantitative Analysis of metabolites were performed using Masshunter Software (Agilent Technologies). The $\mathrm{m} / \mathrm{z}$ values used for quantification were $\mathrm{m} / \mathrm{z} 150.056[\mathrm{M}+\mathrm{H}]^{+}-\left[\mathrm{H}_{2} \mathrm{O}\right]$ for pyridoxal, $\mathrm{m} / \mathrm{z} 130.0503$ $[\mathrm{M}+\mathrm{H}]^{+}-\left[\mathrm{H}_{2} \mathrm{O}\right]$ for glutamate, and $\mathrm{m} / \mathrm{z} 223.074[\mathrm{M}+\mathrm{H}]^{+}$ for cystathionine. Identities were confirmed using an orthogonal characterization (based on exact mass $<10 \mathrm{ppm}$ and on retention time) and MS/MS spectrum, compared with authentic standards (Jové et al. 2011). Data are presented as means \pm standard error from at least three independent experiments. Statistical analysis was performed using the non-parametric Mann-Whitney test.

\section{Results and discussion}

\section{Metabolic disturbances in pyridoxal metabolism in human HD}

Oxidative stress seems to play an important role in HD. In this context, we demonstrated that two enzymes involved in the metabolism of PLP (pyridoxal kinase and antiquitin 1) were oxidized in human HD and also in an HD mouse model (Sorolla et al. 2010). To decipher whether such oxidation may have physiological consequences, we analyzed levels of PDXK in human HD brain samples. Western blot performed with anti-PDXK showed around a $30 \%$ decrease in both striatal (Sorolla et al. 2010) and cortex HD human samples compared to controls (Fig. 1). Decreased levels of PDXK were not unexpected, because it is known that protein oxidation labels them for degradation. PDXK catalyzes pyridoxal phosphorylation and can also phosphorylate both pyridoxine and pyridoxamine, which are further converted to PLP. Thus, PLP synthesis could be impaired both by the decrease in PDXK levels and by its increased oxidation. Antiquitin 1 oxidation observed in HD could result in its inactivation, exacerbating the lack of PLP available as a cofactor. Unfortunately, antiquitin 1 levels could not be detected with confidence by the antibody.

To delve deeper into the effects of PDXK alteration, we used a directed metabolomic approach to analyze whether such PLP deficiency could be detected from a metabolite point of view. Pyridoxal (the substrate of PDXK) appeared clearly identified in the chromatographic separation and its relative quantity was increased in HD human brain (both in cortex and striatum), compared to control subjects (Fig. 2). PLP, the product of the reaction catalyzed by PDXK, could not be detected with confidence probably due to the low levels of PLP compared to pyridoxal (a ratio between $1 / 5$ and 1/10) and more important, to the effect of phosphatases postmortem. 
striatum

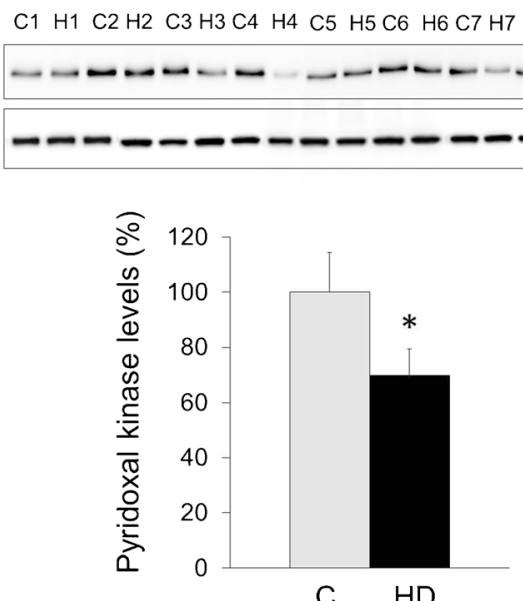

Fig. 1 Levels of PDXK in humans. Western blot analysis against PDXK of tissue extracts were performed. HD (HD) patients and controls (C) samples of human striatum and cortex. Actin was used as a loading control. Bands from the Western blots were quantified and the relative intensities calculated for controls (grey bar) and HD (black bar). The data is represented as a mean relative intensity $\pm \mathrm{SE}$. ${ }^{*} p<0.05$. Striatum data is

\section{Metabolic disturbances in pyridoxal metabolism in R6/1 mice}

Similarly to human results, striatum and cortex samples from 7-month-old R6/1 mice showed decreased levels of PDXK (Fig. 3). When samples from 3-month old mice were analyzed, PDXK also presented lower levels in cortex, but not in striatum (Fig. 3). Cerebellum, a brain area minimally affected by the disease did not show significant differences between R6/1 and control animals (Fig. 3).

Pyridoxal levels were determined in mice samples and similar results from those in humans were obtained in striatum and cortex samples from both 3-month-old and 7-month-old R6/1 mice compared to controls (Fig. 4a). Differences were
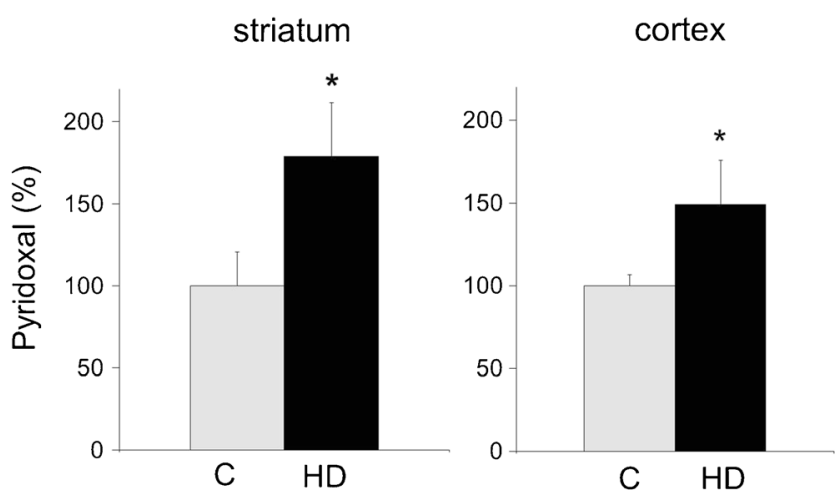

Fig. 2 Levels of pyridoxal in humans. Brain samples were processed and compared with authentic standard. Pyridoxal was quantified in control and HD samples of human striatum and cortex. The data is represented as a mean relative intensity \pm SE. ${ }^{*} p<0.05$ cortex
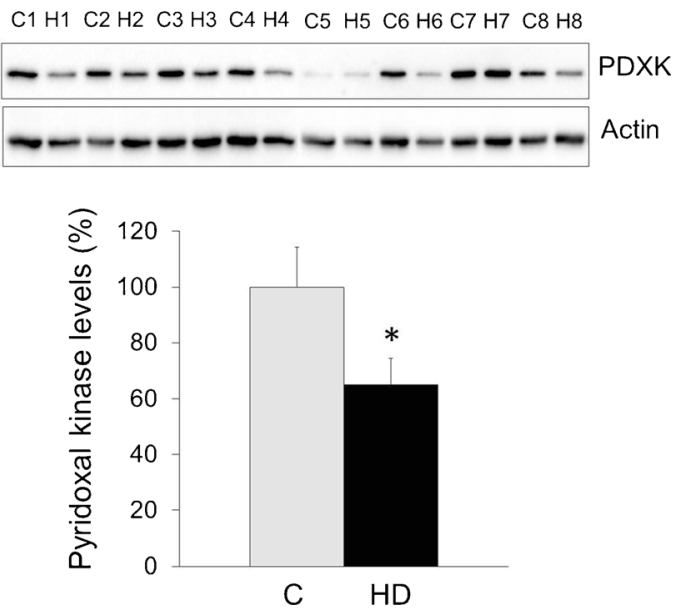

reprinted from Free Radical Biology \& Medicine, 49, Sorolla MA, Rodríguez-Colman MJ, Tamarit J, Ortega Z, Lucas JJ, Ferrer I, Ros J and Cabiscol E. Protein oxidation in Huntington disease affects energy production and vitamin B6 metabolism, 612-621, 2010, with permission from Elsevier

not detected in mouse cerebellum, a brain area not affected by the disease (Fig. 4a). Interestingly, pyridoxal levels were increased as early as 3-month-old R6/1 mice compared to controls. PLP levels were significantly reduced in striatum from 7-month-old R6/1 mice compared to control animals (Fig. 4b). Striatum is the most affected tissue in HD, and 7 month-old animals are in an advanced disease progression stage. Lack of significant differences in cortical PLP levels could be explained by the fact that this area is less affected than striatum in HD. At 3 month-old, PLP levels did not change (Fig. 4b); at this time motor coordination measured by the rotarod performance test was affected but less than at 7-month-old R6/1 mice (not shown). Alteration of pyridoxal levels together with decreased levels of PDXK occurring at early time (3-monthold R6/1 mice) might be primary disturbances in pathogenesis, triggering a decrease in PLP levels in a later stage, when motor coordination is affected. All these evidences suggest that primarly impaired pyridoxal metabolim leads finally to PLP deficiency in striatum, a motor control area, which might act as an event for motor disturbances in HD.

\section{Glutamate and cystathionine are increased in HD}

PLP deficiency in HD might affect glutamate decarboxylase (GAD), a PLP-dependent enzyme. It has been postulated that GAD activity is regulated by a cycle of activation and inactivation by the binding or realease of its cofactor (Battaglioli et al. 2003) and GAD activity is compromised in vivo by diminished levels of PLP in rat forebrain homogenates in an age dependent manner (Massieu et al. 1994). Such impairment 
Fig. 3 Levels of PDXK in mice. Western blot analysis against PDXK of tissue extracts were performed. WT (C) and R6/1 (H) mice from striatum, cortex, and cerebellum areas (3M: 3-monthsold mice; 7M: 7-month-old mice). Actin was used as a loading control. Bands from the Western blots were quantified and the relative intensities calculated for controls (grey bar) and HD (black bar). The data is represented as a mean relative intensity $\pm \mathrm{SE}$. ${ }^{*} p<0.05$ striatum

cortex

cerebellum

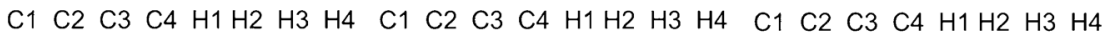
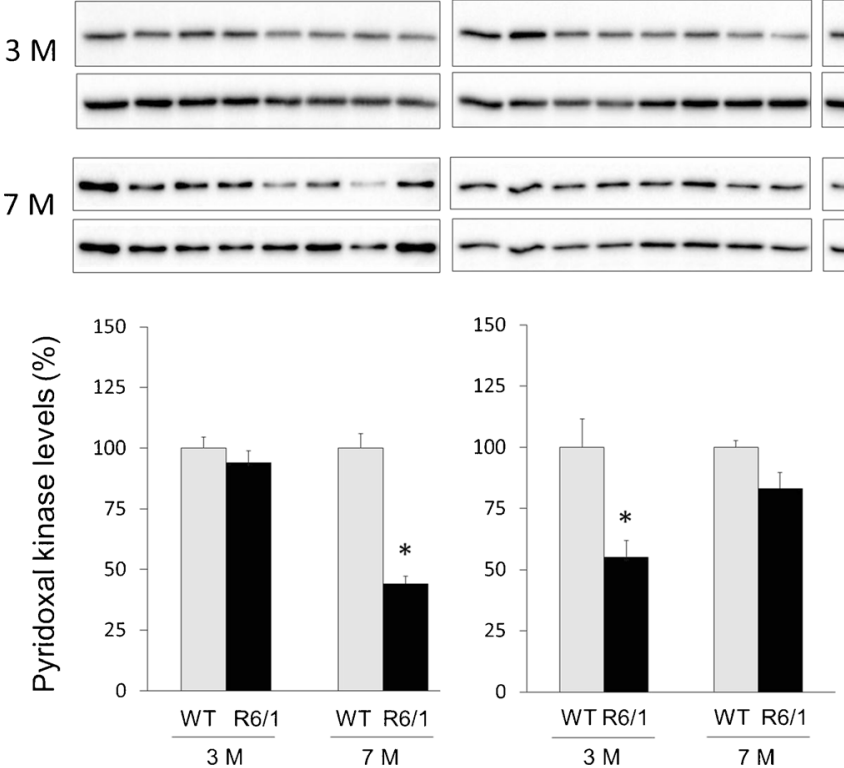

in catalytic activity of glutamate decarboxylase would result in increased levels of glutamate and decreased levels of GABA, the product of the reaction. In humans, glutamate levels presented a high variability between individuals, precluding the observation of any statistically significant differences between HD and controls (not shown). In mice, glutamate was clearly identified as being increased in all 3 studied tissues from 7-month-old R6/1 mice compared to WT
Fig. 4 Levels of pyridoxal and PLP in mice: a Pyridoxal, and $\mathbf{b}$ PLP were quantified in WT and R6/1 mice from striatum, cortex, and cerebellum areas (3M: 3 month-old mice; 7M: 7-monthold mice). The data is represented as a mean relative intensity \pm SE. $* p<0.05$
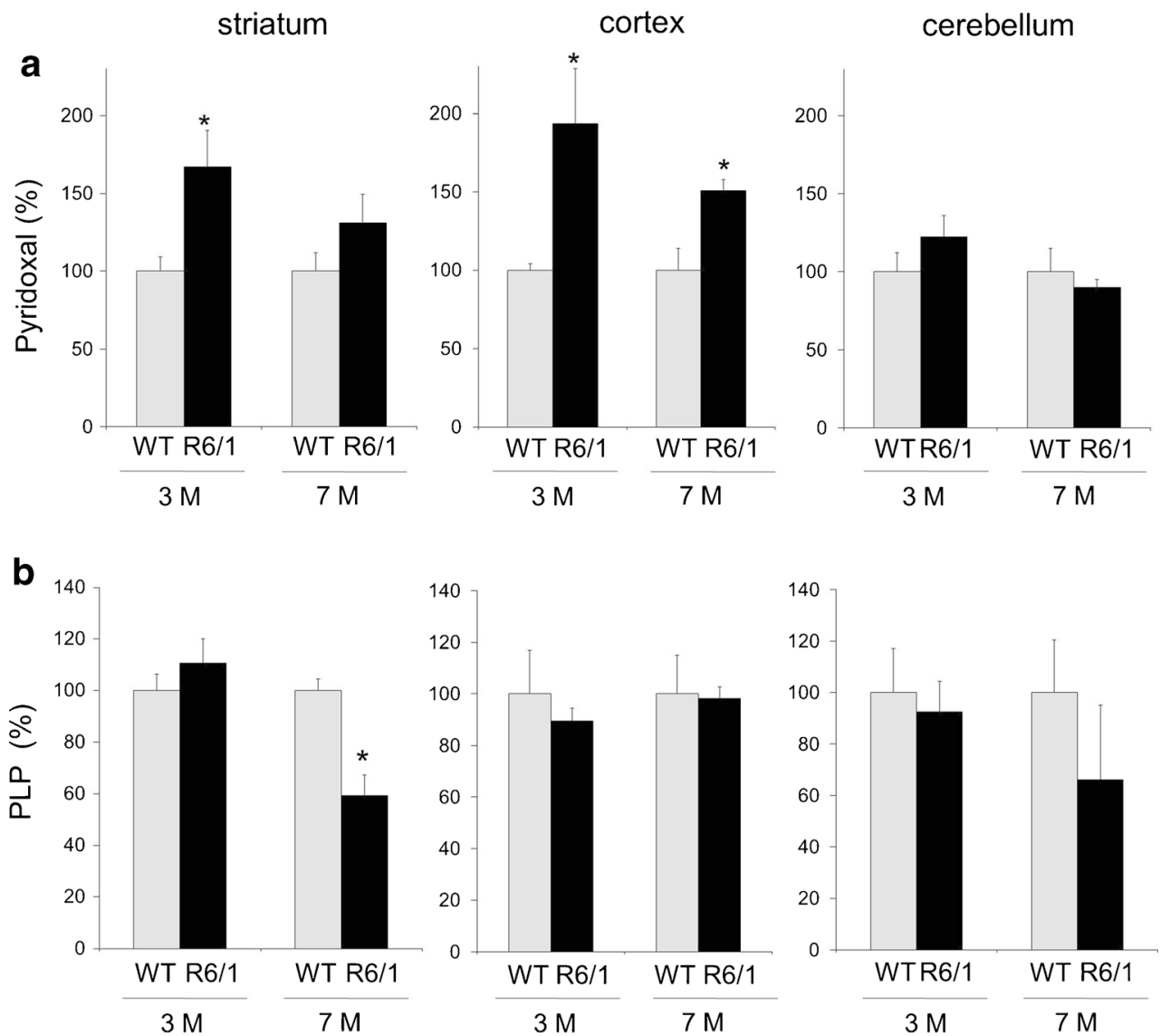
(Fig. 5a). Increases in glutamate levels were only statistical significant in striatum and cortex and greater in these tissues than in cerebellum. In 3-month-old mice, although glutamate levels were slightly higher in R6/1 mice, no statistically significant differences were detected (not shown). Such observations are in agreement with the proposal that an excess of glutamate is the mechanism for cell death in the excitotoxic model of HD, especially in striatum (Tabrizi et al. 1999; Anitha et al. 2011). GABA levels could not be detected in our samples. Nevertheless, in human postmortem HD studies, the concentration of this neurotransmitter in HD has been described to be reduced to less than one-third of normal values in the striatum (Perry et al. 1973), with deficits exceeding $25 \%$ in the cortex (Reynolds and Pearson 1987). Similarly, striatum-specific lower concentrations of GABA are described in the R6/1 mouse model (Tsang et al. 2006) and in rats (Tsang et al. 2009). These results are important since striatal neurodegeneration is reflected by intrinsic neuron deficiency in the neurotransmitters, particularly in GABA. Our results may explain why 2 years of glutamate administration in HD patients (the substrate for glutamate decarboxylase) and pyridoxine did not improve motor and behavioral function (Barr et al. 1978). Therefore, we would hypothesize that pyridoxine administration was not efficiently converted to PLP due to PDXK deficiency and that decreased PLP

Fig. 5 Levels of glutamate and cystathionine in mice. Brain samples of 7-month-old WT and R6/1 mice were processed and compared with authentic standards. a Glutamate, and $\mathbf{b}$ cystathionine were quantified from striatum, cortex, and cerebellum areas. The data is represented as a mean relative intensity \pm SE. ${ }^{*} p<0.05$
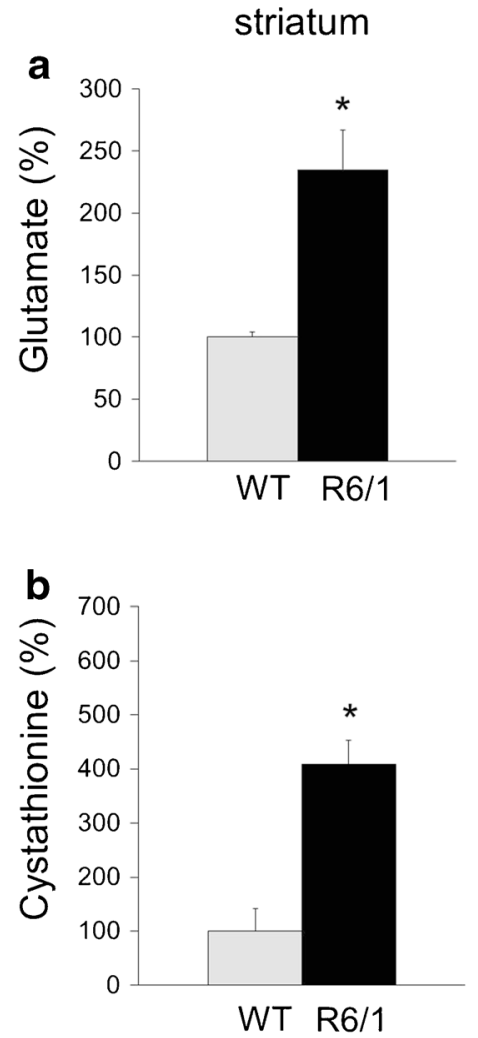

availability would inhibit GABA production by glutamate decarboxylase.

Glutathione metabolism might also be affected by PLPdeficiency. Cystathionine is converted to cysteine (a precursor of glutathione) by cystathionine gamma-ligase, which uses PLP as a cofactor. In humans, cystathionine levels did not show statistically significant differences between HD and controls due to high individual variability (not shown). However, cystathionine levels were highly increased in all three $7-$ month-old R6/1 tissues compared to control animals (Fig. 5b), which could be due to a deficit in cystathionine gamma ligase activity. Cystathionine could not be detected in young mice. According to our hypothesis, lower levels of PLP would also affect GSH synthesis and, as a consequence, the entire GSH-dependent antioxidant defense system. However, we found no significant differences in GSH levels either in human or in mouse samples (not shown). Published results are not conclusive. Altered glutathione metabolism was described using metabolomic approaches in 3nitropropionic treated rats (Chang et al. 2011). In HD patients, lower levels of reduced GSH were detected both in plasma (Klepac et al. 2007) and in red blood cells (Zanella et al. 1980). Nevertheless, increased total glutathione levels were observed in cortical and striatal mitochondria of R6/2 mice (Choo et al. 2005). Postmortem oxidation of GSH to GSSG is a key factor that may explain such variability between published studies.
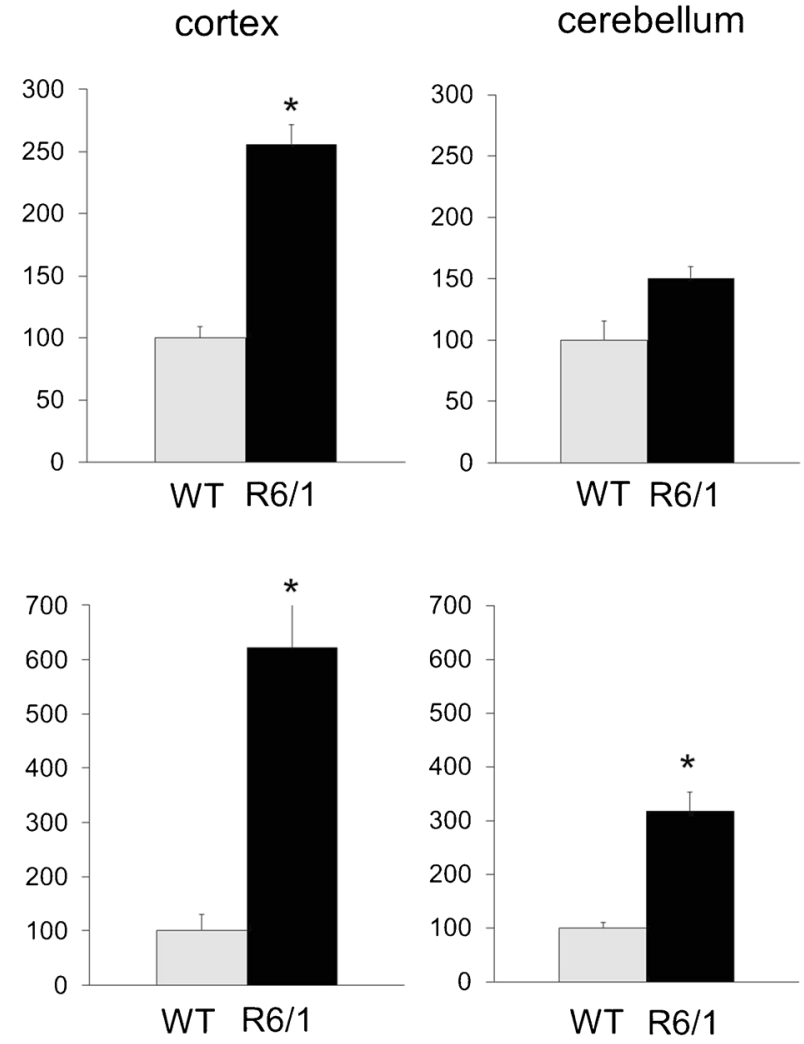


\section{Conclusions}

The existence of oxidative stress in affected areas of HD patients and animal models is widely assumed. Although it is not completely understood how mHtt causes this stress, a toxic gain of function is well accepted. It is clear that $\mathrm{mHtt}$ expression results in increased levels of oxidatively damaged proteins in specific areas of the HD-affected brain (Sorolla et al. 2012). Oxidation of enzymes like PDXK might explain the decreased levels of this enzyme observed in human and murine HD samples. In agreement, pyridoxal levels were increased both in striatum and cortex and PLP was found to be reduced in striatum. The fact that in cerebellum, a less affected area in HD, differences were not detected underline their relevance in HD. Decreased PLP-dependent metabolism would affect important pathways known to be altered in HD, including synthesis of neurotransmitters like GABA and GSH metabolism. In this context, treatment with PLP, the active form of vitamin B6 (instead of pyridoxine or pyridoxal), should be considered for evaluation as a potential therapy.

Acknowledgments Metabolomic analysis has been performed in the Metabolomic Facility of the Universitat de Lleida/IRBLleida. We are indebted to Mariona Jové, Ph.D., for a helpful discussion of the manuscript. This work has been supported by grant BFU2010-17387 from the Ministerio de Educación y Ciencia (Spain). We thank Elaine M. Lilly, Ph.D., for editing assistance.

\section{Compliance with ethical standards}

Conflict of interest Authors declare that they have not conflict of interest.

Research involving participants (post-mortem samples) and/or animals All procedures performed in studies involving human participants were in accordance with ethical standards of the institutional and/or national research committee and with the 1964 Helsinki declaration and its later amendments or comparable ethical standards.

All applicable international, national and institutional guidelines for the care and use of animals were followed. All procedures performed in studies involving animals were in accordance with the ethical standards of the institution or practice at which the studies were conducted.

Informed consent Informed consent was obtained from the families of all individual participants in the study.

\section{References}

Anitha M, Nandhu MS, Anju TR, et al. (2011) Targeting glutamate mediated excitotoxicity in Huntington's disease: neural progenitors and partial glutamate antagonist-memantine. Med Hypotheses 76:138140. doi:10.1016/j.mehy.2010.09.003

Arrasate M, Mitra S, Schweitzer ES, et al. (2004) Inclusion body formation reduces levels of mutant huntingtin and the risk of neuronal death. Nature 431:805-810 http://www.nature.com/nature/journal/ v431/n7010/suppinfo/nature02998_S1.html
Barr AN, Heinze W, Mendoza JE, Perlik S (1978) Long term treatment of huntington disease with L-glutamate and pyridoxine. Neurology 28 : $1280-1282$

Battaglioli G, Liu H, Martin DL (2003) Kinetic differences between the isoforms of glutamate decarboxylase: implications for the regulation of GABA synthesis. J Neurochem 86(4):879-887. doi:10.1046/j. 1471-4159.2003.01910.x

Benitez J, Fernandez E, Ruiz PG, et al. (1994) Trinucleotide (CAG) repeat expansion in chromosomes of Spanish patients with Huntington's disease. Hum Genet 94:563-564. doi:10.1007/ BF00211028

Browne SE (2008) Mitochondria and Huntington's disease pathogenesis: insight from genetic and chemical models. Ann N Y Acad Sci 1147: 358-382. doi:10.1196/annals.1427.018

Chang KL, New LS, Mal M, et al. (2011) Metabolic profiling of 3nitropropionic acid early-stage Huntington's disease rat model using gas chromatography time-of-flight mass spectrometry. J Proteome Res 10:2079-2087. doi:10.1021/pr2000336

Choo YS, Mao Z, Johnson GVW, Lesort M (2005) Increased glutathione levels in cortical and striatal mitochondria of the R6/2 Huntington's disease mouse model. Neurosci Lett 386:63-68. doi:10.1016/j. neulet.2005.05.065

DiFiglia M, Sapp E, Chase KO, et al. (1997) Aggregation of huntingtin in neuronal intranuclear inclusions and dystrophic neurites in brain. Science 277:1990-1993. doi:10.1126/science.277.5334.1990

Dugan LL, Sensi SL, Canzoniero LM, et al. (1995) Mitochondrial production of reactive oxygen species in cortical neurons following exposure to N-methyl-D-aspartate. J Neurosci 15:6377-6388

Group THDCR (1993) A novel gene containing a trinucleotide repeat that is expanded and unstable on Huntington's disease chromosomes. The Huntington's Disease Collaborative Research Group. Cell 72: 971-983

Jenkins BG, Koroshetz WJ, Beal MF, Rosen BR (1993) Evidence for impairment of energy metabolism in vivo in Huntington's disease using localized 1 H NMR spectroscopy. Neurology 43:2689-2695

Jové M, Serrano JCE, Ortega N, et al. (2011) Multicompartmental LC-QTOF-based metabonomics as an exploratory tool to identify novel pathways affected by polyphenol-rich diets in mice. J Proteome Res 10:3501-3512. doi: $10.1021 / \mathrm{pr} 200132 \mathrm{~s}$

Klepac N, Relja M, Klepac R, et al. (2007) Oxidative stress parameters in plasma of Huntington's disease patients, asymptomatic Huntington's disease gene carriers and healthy subjects : a crosssectional study. J Neurol 254:1676-1683. doi:10.1007/s00415007-0611-y

Mangiarini L, Sathasivam K, Seller M, et al. (1996) Exon 1 of the HD gene with an expanded CAG repeat is sufficient to cause a progressive neurological phenotype in transgenic mice. Cell 87:493-506

Massieu L, Rivera A, Tapia R (1994) Convulsions and inhibition of glutamate decarboxylase by pyridoxal phosphate-gamma-glutamyl hydrazone in the developing rat. Neurochem Res 19(2):183-187

Mills PB, Struys E, Jakobs C, et al. (2006) Mutations in antiquitin in individuals with pyridoxine-dependent seizures. Nat Med 12:307309. doi:10.1038/nm1366

Perry TL, Hansen S, Kloster M (1973) Huntington's chorea. Deficiency of gamma-aminobutyric acid in brain. N Engl J Med 288:337-342. doi:10.1056/nejm197302152880703

Perutz MF (1999) Glutamine repeats and neurodegenerative diseases: molecular aspects. Trends Biochem Sci 24:58-63. doi:10.1016/ S0968-0004(98)01350-4

Rankin PM, Harrison S, Chong WK, et al. (2007) Pyridoxine-dependent seizures: a family phenotype that leads to severe cognitive deficits, regardless of treatment regime. Dev Med Child Neurol 49:300-305. doi:10.1111/j.1469-8749.2007.00300.x

Reiner A, Albin RL, Anderson KD, et al. (1988) Differential loss of striatal projection neurons in Huntington disease. Proc Natl Acad Sci U S A 85:5733-5737 
Reynolds GP, Pearson SJ (1987) Decreased glutamic acid and increased 5-hydroxytryptamine in Huntington's disease brain. Neurosci Lett 78:233-238

Shirendeb U, Reddy AP, Manczak M, et al. (2011) Abnormal mitochondrial dynamics, mitochondrial loss and mutant huntingtin oligomers in Huntington's disease: implications for selective neuronal damage. Hum Mol Genet 20:1438-1455. doi:10.1093/hmg/ddr024

Sorolla MA, Reverter-Branchat G, Tamarit J, et al. (2008) Proteomic and oxidative stress analysis in human brain samples of Huntington disease. Free Radic Biol Med 45:667-678. doi:10.1016/j. freeradbiomed.2008.05.014

Sorolla MA, Rodríguez-Colman MJ, Tamarit J, et al. (2010) Protein oxidation in Huntington disease affects energy production and vitamin B6 metabolism. Free Radic Biol Med 49:612-621. doi:10.1016/j. freeradbiomed.2010.05.016

Sorolla MA, Rodríguez-Colman MJ, Vall-Llaura N, et al. (2012) Protein oxidation in Huntington disease. Biofactors 38:173-185. doi:10. 1002/biof.1013
Tabrizi SJ, Cleeter MW, Xuereb J, et al. (1999) Biochemical abnormalities and excitotoxicity in Huntington's disease brain. Ann Neurol 45: 25-32 ISSN: 0364-5134

Tsang TM, Woodman B, McLoughlin GA, et al. (2006) Metabolic characterization of the R6/2 transgenic mouse model of Huntington's disease by high-resolution MAS 1H NMR spectroscopy. J Proteome Res 5:483-492. doi:10.1021/pr050244o

Tsang TM, Haselden JN, Holmes E (2009) Metabonomic characterization of the 3-nitropropionic acid rat model of Huntington's disease. Neurochem Res 34:1261-1271. doi:10.1007/s11064008-9904-5

Vonsattel JP, Myers RH, Stevens TJ, et al. (1985) Neuropathological classification of Huntington's disease. J Neuropathol Exp Neurol 44:559-577

Zanella A, Izzo C, Meola G, et al. (1980) Metabolic impairment and membrane abnormality in red cells from Huntington's disease. J Neurol Sci 47:93-103 